\title{
Formulation and Evaluation of Novel Herbal Aerosol for Arthritis
}

\author{
Rajasekaran Aiyalu ${ }^{1 *}$, Ithyaventhan Subramaniam ${ }^{2}$, Arulkumaran Govindarajan ${ }^{1}$ and \\ Arivukkarasu Ramasamy ${ }^{1}$ \\ ${ }^{1} \mathrm{KMCH}$ College of Pharmacy, Coimbatore, Tamilnadu, India \\ ${ }^{2}$ Doctor Thangs Products, Coimbatore, Tamilnadu, India
}

Received: May 15, 2017; Accepted: May 22, 2017; Published: December 27, 2017

*Corresponding author: Rajasekaran Aiyalu, KMCH College of Pharmacy, Kovai Estate, Kalapatti Road, Coimbatore - 641048, Tamilnadu, India, Tel: +91-422-2369301; Fax: +91-422-2369302; E-mail: rsekaran2001in@yahoo.co.in

\begin{abstract}
Arthritis is the most common cause of disability in adults all over the world, limiting everyday activities for billions of people. Herbs have been paid more attention since they have very little side effects. Cardiospermum halicacabum Linn, (Sapindaceae) and VitexnegundoLinn. (Verbenaceae) were used in traditional medicine for the treatment of arthritis. The present investigation was undertaken to evaluate anti-arthritic activity of novel herbal aerosol spray formulation containing methanol leaf extracts of Cardiospermum halicacabum (MLECH) and Vitexnegundo (MLEVN). A novel topical aerosol spray was formulated using MLECH and MLEVN $(2 \% \mathrm{w} / \mathrm{v}$ each) and their quality control, stability and efficacy studies was carried out as per United States Pharmacopoeia (USP), International Conference on Harmonization (ICH) and Organization for Economic Co-Operation and Development (OECD) guidelines respectively. CFA induced arthritis method was employed for the evaluation of antiarthritic activity. Assessment of body weight, biochemical parameters, hematological parameters, serum biomarker levels, photographic, radiographic analysis and histopathological investigations were carried out as a supportive evidence for the anti-arthritic efficacy of the developed novel herbal aerosol spray formulation. The results of the quality control test complied with USP standards and the stability study clearly revealed that the formulated topical herbal aerosol spray was found to be stable. Significant $(p<0.01)$ alterations in rat paw volume, body weight, biochemical, hematological, serum biomarker levels and reduction in inflammation and hyperplasia of synovium of formulation treated rats were observed compared with arthritic rats. Study concluded that the formulated novel herbal aerosol spray exhibited good anti-arthritic activity.
\end{abstract}

Keywords: CFA induced arthritis; Rheumatoid Factor; TNF $\alpha$; herbal aerosol

\section{Introduction}

Arthritis is a disease condition that affects the musculoskeletal system, causing painful inflammation and stiffness around joints. In Greek "artho" means joint and "itis" means inflammation and so arthritis is a form of joint disorder that involves inflammation of joints. Among the 100 different forms of arthritis, the most common forms are osteoarthritis, Rheumatoid Arthritis (RA), psoriatic arthritis and related autoimmune disease, septic arthritis caused mainly by joint infection. There is no specific drug available for the treatment of arthritis and hence pharmaceutical companies continuing their research for the discovery and development of a drug specifically for RA. However, steroidal, non-steroidal anti-inflammatory, Disease Modifying Antirheumatic Drugs (DMARDs) and immunosuppressant drugs are being most commonly prescribed for the treatment of RA. Though the goal of these drugs is to relieve pain and to decrease joint inflammation, to prevent joint destruction and to restore function of disabled joints, these drugs are known to produce adverse side effects [1]. Most notable adverse effects produced by NSAIDs are gastric erosions, ulceration, bleeding, perforation [2] and acute renal failure [3]. Serious and fatal liver toxicity was reported for DMRADs [4]. Therefore, reducing side effects should be considered while designing a formulation with improved therapeutics for RA. The Siddha and Ayurvedic systems of treatment are being increasingly recognized as an alternate approach to arthritic treatment. Besides, investigation of the efficacy of plant based drugs used in the traditional medicine has been paid more attention since they are cheap, readily available and have very little side effects. In view of this fact, an attempt was made to develop an alternative or traditional medicine for the treatment of arthritis by the herbs used in traditional practice. Since $\mathrm{CH}$ and VN were most commonly used in traditional practice for the treatment of arthritis, they were selected for the study.

Cardiospermum Halicacabumlinn $(\mathrm{CH})$ is a climber belongs to the family Sapindaceae locally known as balloon vine and called as "modakathon" in Tamil. "Modaku" means crippling joint pain; "thon" means remedy. It is a deciduous, branched, herbaceous climber distributed throughout the plains of India. $\mathrm{CH}$ reported to contain arachidic acid, apigenin, apigenin-7-0-glucuronide, chysoeriol-7-0-glucuronide, luteolin, luteolin-7-0-glucuronide, saponin, quebrachitol, proanthocyanidin, beta sitosterol and stigmosterol [5]. Irulas in Thanjavur, Tamilnadu, India, provided evidence for the use of this plant as a traditional remedy that has been used for centuries to treat RA and it is still used by some locals to treat RA in Asian [6] communities, substantiated by many researchers [7-10]. Anti-inflammatory properties of $\mathrm{CH}$ have 
been researched [11], but no clinical trials have been reported so far. Experimental pharmacological studies of $\mathrm{CH}$ have shown antioxidant activity [12] analgesic and vasodepressant activities [13], antipyretic activity [14], Antimalarial activity [15], diuretic activity [16], anti-diarrheal activity [17], anti-diabetic activity [18] and anti-ulcer activity [19].

VitexnegundoLinn (VN) (Verbenaceae), grows gregariously in wastelands and is also planted as a hedge-plant. It was reported for the presence phytochemical constituents, flavonoids such as casticin, orientin, isoorientin, luteolin, lutecin-7-0-glucoside, corymbosin; gardenins A and B [20]. VN has been documented for potent anti-inflammatory [21], anti-arthritic [22], anticonvulsant [23], hepatoprotective [24], laxative [25] and bronchial relaxant activities [26]. Though all parts of $\mathrm{CH}$ and $\mathrm{VN}$ are used, leaves are majorly used for treating arthritis in traditional medicine [27] and hence a dosage form was designed using the leaf extracts of the plants.

The existing topical formulations available have the disadvantages viz. difficulty in applying the formulation to the respective site, staining cloth due to greasy nature, more chance of contamination and more wastage due to inaccuracy of dose. The designed herbal aerosol spray formulation have the advantages viz. ease of administration, cause no contamination, no clean up procedure after using the product, protection of active constituents from oxygen and moisture, minimum pain and irritation experience during application, minimum side effects as it is a herbal medicine, stable as the constituents are enclosed in a pressurized container, rapid onset of action, accuracy of the administered dose, no first pass effect and GIT degradation and can be delivered directly on to the affected area. Further, exhaustive work on $\mathrm{CH}$ and VN has been done for its biological activity and mechanism of action through systemic application. But there is no report on preclinical studies of $\mathrm{CH}$ and $\mathrm{VN}$ for its anti-arthritic activity by topical application. Thus, keeping all this in view, a topical herbal aerosol spray was developed using MLECH and MLEVN and evaluated for anti-arthritic activity.

\section{Materials and Methods}

\section{Plant Materials and Chemicals}

Both the plants $\mathrm{CH}$ and VN were collected in November 2010 from hilly area of Palakkad district, Kerala, India. Plants were authenticated by GVS. Murthy, Director of Botanical survey of India, Coimbatore, India and the Voucher specimens were deposited at KMCH College of Pharmacy, Coimbatore. Apigenin, carrageenan, Complete Freund's adjuvant (CFA), Diclofenac sodium and luteolin purchased from Sigma Aldrich, USA was used for study. Solvents were procured from Qualigens, India. Aerosol aluminum containers fitted with valve assembly and propellants were procured from Aspire industries, Mumbai, India.

\section{Preparation of Extracts}

Fresh mature leaves of $\mathrm{CH}$ and $\mathrm{VN}$ was cleaned and then shade dried at room temperature $(32 \pm 20 \mathrm{C})$ for 10 days, pulverized to coarse powder, passed through a \# 40 mesh sieve and extracted separately with methanol at 660C in a soxhlet apparatus for 72h. Each of the solvent extracts was filtered and concentrated separately under reduced pressure with IKA Rotary evaporator (Model No RN 10 digital V, ILMAC Germany) at 400C.

\section{Preparation of Topical Herbal Aerosol Spray Formulation}

Aerosol dosage form was formulated using, the product concentrate $(2 \% \mathrm{w} / \mathrm{v}$ of extract each of Cardiospermum halicacabum and Vitexnegundo containing $0.02 \% \mathrm{w} / \mathrm{v}$ of sodium benzoate and the propellant (mixture of propane and butane). The product concentrate was quantitatively packed to the cold sterilized aerosol container, the valve assembly was inserted into the container and crimped using semi automatic aerosol filling machine (Twin Tech, India). Then the propellant was filled into the container using automatic aerosol filling machine. Packed containers passed through a quality check for leakage followed by labeling and stamping of batch number and manufacturing date.

\section{Quality Control of Topical Herbal Aerosol Spray Formulation}

The quality control tests viz. flammability test, physicochemical characterization, performance and biological evaluation for the finished topical herbal aerosol spray formulation were evaluated as per the procedure described in Lachman et al [28] and USP [29].

\section{Stability Studies of Topical Herbal Aerosol Spray Formulation}

The stability study for the topical herbal aerosol spray formulation was done as per ICH guidelines in a stability chamber for a period of 6 months. The selected topical herbal aerosol spray formulation consisting of $2 \%$ of each MECH and MEVN were loaded in a humidity chamber $\left(300 \mathrm{X} 300 \mathrm{X} 300 \mathrm{~mm}, 15-60^{\circ} \mathrm{C}\right.$, Technico, India) at $25^{\circ} \mathrm{C} \pm 2{ }^{\circ} \mathrm{C} / 60 \% \mathrm{RH} \pm 5 \% \mathrm{RH}, 32^{\circ} \mathrm{C} \pm 2{ }^{\circ} \mathrm{C} / 60 \%$ $\mathrm{RH} \pm 5 \% \mathrm{RH}$ and $40^{\circ} \mathrm{C} \pm 2{ }^{\circ} \mathrm{C} / 75 \% \mathrm{RH} \pm 5 \% \mathrm{RH}$. Samples were withdrawn at an initial, first, second, third and sixth months and evaluated for change in color, odor, weight, pH, density, viscosity, net content, leakage test, microbial load and sterility test and the observations were recorded in Table 2.

\section{Animals}

Wistar strains rats of either sex were obtained from the animal house of Kovai medical center research and educational trust, Coimbatore, India. The animals were kept in polypropylene cages at a temperature of $25 \pm 2^{\circ} \mathrm{C}$, humidity $(50 \pm 5) \mathrm{RH}$ and 12 $\mathrm{h}$ light and dark cycles. They were fed with standard laboratory animal diet and water ad libitum. Animals were acclimatized to laboratory conditions before the test. Experiments were designed and conducted in accordance with ethical norms approved by Committee for the Purpose of Control and Supervision on Experiments on Animals (CPCSEA) and Institutional Animal Ethical Committee (KMCRET/DST/01/2011, dt.16/07/2011).

\section{Anti-Arthritic Activity}

Efficacy of the topical herbal aerosol spray formulation was 
studied by CFA induced arthritis model [30] in rats through oral administration and topical application. The rats were divided into six groups, each consisting of six animals. Group 1 received ( $1 \mathrm{ml}) 1 \% \mathrm{w} / \mathrm{v}$ of Sodium Lauryl Sulphate (SLS) was considered as normal control. Arthritis was induced to group 2 to 6 by injecting a $0.1 \mathrm{ml}(0.1 \% \mathrm{w} / \mathrm{v})$ suspension of killed Mycobacterium tuberculosis bacteria (Genei, Bangalore) homogenized in liquid paraffin into the left hind foot in the subplantar region of rats. Groups (2 to 6) administered with CFA were allowed to develop arthritis for 21 days. During the experimental period, body weight of the animals and the rat paw volume of control and treatment groups were measured on $4^{\text {th }}, 8^{\text {th }}, 14^{\text {th }}$ and $21^{\text {st }}$ day by using digital Vernier caliper (Mitetoyodigimatic caliper, Japan).

The overnight fasted animals were anaesthetized with ketamine $(20 \mathrm{mg} / \mathrm{kg}$, i.p) followed by withdrawal of blood samples from retro-orbital sinus and the collected blood samples were evaluated for hematological parameters. A portion of the blood samples were centrifuged at $10000 \mathrm{rpm}$ for $10 \mathrm{~min}$. The separated serum was analyzed for C-reactive protein (CRP) and Rheumatoid Factor (RF). Photographic and radiographic analysis was carried out using ALLANS fully computerized X-ray instrument (AXD-100i, Mumbai, India) for the confirmation of development of arthritis.

After the confirmation of arthritis development, Diclofenac sodium $10 \mathrm{mg} / \mathrm{kg}$ (p.o.) and the combined doses of MLECH and MLEVN (400 mg/kg each) (p.o.) were administered orally from 22 to 42 days to Group 3 (served as reference standard for oral route) and Group 4 respectively. Topical Diclofenac Diethylamine aerosol spray (8 $\mathrm{mg} /$ day) and topical herbal aerosol spray formulation $(64 \mathrm{mg} /$ day) were sprayed continuously for $3 \mathrm{sec}$ from 22 to 42 days to the left knee joint region of Group 5 (served as reference standard for topical route) and Group 6 respectively. During the treatment period, the body weight of the animals and the rat paw volume of control and treatments were measured on 25th, 29th, 35th and 42nd day by using digital Vernier caliper (Mitetoyo digimatic caliper, Japan). At the end of 42nd day the pain test score of the animals were recorded visually31. The overnight fasted animals were anaesthetized with ketamine (20 $\mathrm{mg} / \mathrm{kg}$, i.p) followed by withdrawal of blood samples from retroorbital sinus and the collected blood samples were evaluated for hematological parameters [32].A portion of the blood samples were centrifuged at $10000 \mathrm{rpm}$ for $10 \mathrm{~min}$. The separated serum was analyzed for urea, uric acid and serum biomarkers viz. CRP, RF (Omega diagnostics Limited, Scotland, UK), TNF $\alpha$ (ELISA Kit, Gen-Probe, France), IL-1ß and IL6 (ELISA Kit, R\&D systems, USA). Biochemical investigations were carried out in a Autoanalyer (Photometer 5010 V5+, Robert Riely, Berlin) using Piramal healthcare limited reagent kit.

Photographic and radiographic analysis was carried out at the end of the arthritis experiment using ALLANS fully computerized X-ray instrument (AXD-100i, Mumbai, India) for the assessment of reduction in arthritis (Figures1A and 1B). The animals were sacrificed by cervical dislocation and the organs thymus, spleen and bone joints of ankle joint were isolated, weighed and [33] observed for histopathological changes under microscope and digitalimages were acquired [Figures1A and 1B].

\section{$10 \mathrm{X}$}

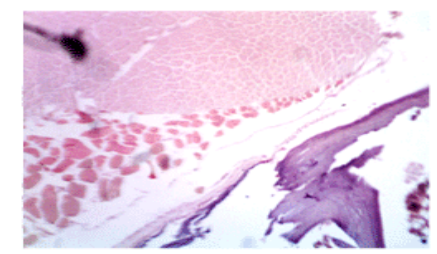

$40 X$

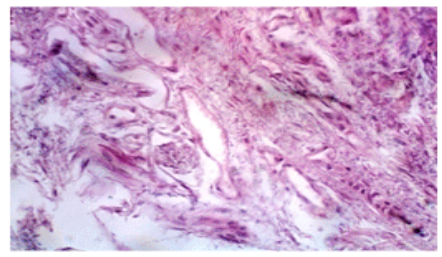

Normal control showing normal adjacent soft tissue and synovium (a)
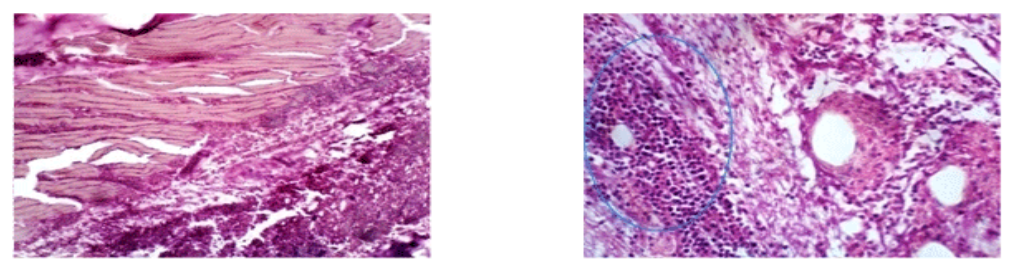

CFA control showing adjacent soft tissue and synovial hyperplasia with severe inflammation (b)
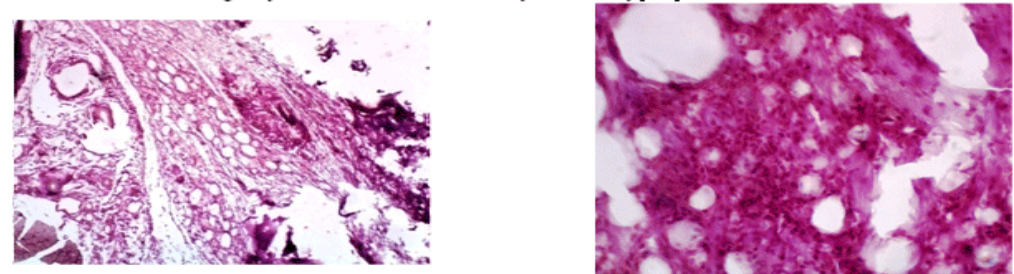

CFA \& diclofenac treated (p.o.) showing occasional inflammatory cells \& hyperp lasia of the synovium (c)

Figure 1A:Histopathological sections of ankle joint of (a) normal rat (b) arthritic control rats (c) diclofenac sodium treated rats (p.o.) under $10 \mathrm{X}$ and $40 \mathrm{X}$ magnification 

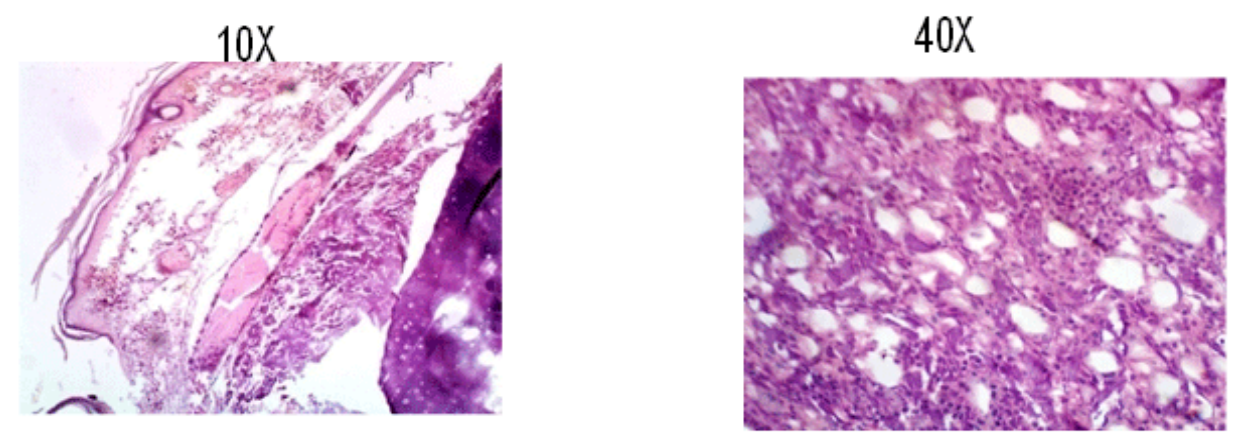

CFA \& MLECH +MLEVN treated (p.o.) showing mild inflammation \& hyperplasia of the

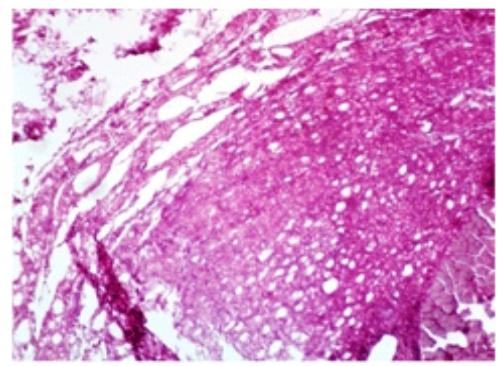

synovium (d)

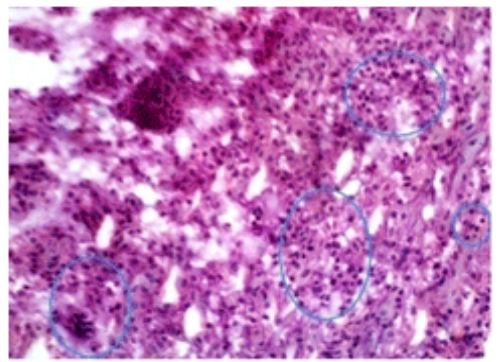

CFA \& diclofenac aerosol spray treated showing moderate inflammation \& hyperplasia of the

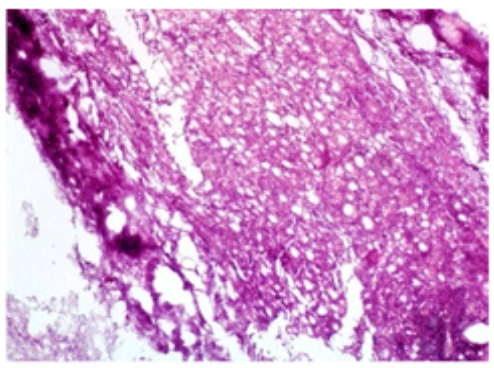

synovium (e)

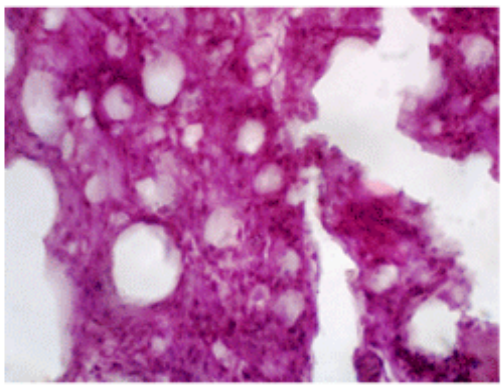

CFA \& topical herbal aerosol spray showing occasional inflammatory cells \& hyperplasia of the synovium (f)

Figure 1B: Histopathalogical sections of ankle joint of (d) MLECH and MLEVN (400+400 mg/kg) treated rats (p.o.) (e)diclofenacdiethylamine topical aerosol spray treated rats (f) topical herbal aerosol spray formulation treated rats under $10 \mathrm{X}$ and $40 \mathrm{X}$ magnification.

\section{Statistical Analysis}

The results are expressed as the mean \pm SEM. The significance of the difference was evaluated by one-way ANOVA followed by Dunnett's test. Data were considered statistically significant if $\mathrm{p}$ $<0.05$.

\section{Results}

\section{Quality Control Test for Topical Herbal Aerosol Spray Formulation}

In flammability test the flame extended for $6 \mathrm{~cm}$ after spraying the formulation in open flame and flame extension test confirms that the product is not extremely flammable. The results of physico chemical characterization viz. vapour pressure, density, $\mathrm{pH}$, viscosity, compatibility of product concentrates with propellants, discharge rate, spray pattern, Net content, particle size, leakage test and spray angle complied with the standards of USP [Table 1].No edema or redness of skin was observed in rabbits after topical administration of the aerosol spray formulation.

\section{Stability Studies of Topical Herbal Aerosol Spray Formulation}

There is no change in color, odour, ph, density, viscosity, net content and leakage of the topical herbal aerosol spray formulation were examined at 0,1,2,3 and 6 months [Table 2]. Thus the results of the study clearly revealed that the formulated topical aerosol is found to be stable. 
Table 1: Quality Control test for topical herbal aerosol spray formulation

\begin{tabular}{|c|c|c|c|c|}
\hline S.No & & Parameters & Value & Units \\
\hline 01 & Flammability & Flame Extension & 6 & $\mathrm{~cm}$ \\
\hline \multirow{5}{*}{02} & \multirow{5}{*}{$\begin{array}{l}\text { Physico chemical } \\
\text { characteristics }\end{array}$} & Vapour Pressure & 160 & psig \\
\hline & & Density & 0.851 & $\mathrm{~g}$ \\
\hline & & $\mathrm{pH}$ & $4.20-4.33$ & -- \\
\hline & & Viscosity & 0.695 & centipoise \\
\hline & & $\begin{array}{l}\text { Compatibility of product concentrates } \\
\text { with propellants }\end{array}$ & \multicolumn{2}{|c|}{ No turbidity was observed } \\
\hline \multirow{7}{*}{03} & \multirow{7}{*}{ Performance } & Aerosol valve discharge rate & 0.284 & $\mathrm{~g} / 3 \mathrm{sec}$ \\
\hline & & Spray pattern & 6 & $\mathrm{~cm}$ \\
\hline & & Net content & 84 & g \\
\hline & & Leakage test & \multicolumn{2}{|c|}{ No leakage was observed } \\
\hline & & Particle size range & $13-60$ & $\mu \mathrm{m}$ \\
\hline & & $\begin{array}{l}\text { Total number of discharges per } \\
\text { container }\end{array}$ & 98 puff & $3 \mathrm{sec}$ for each puff \\
\hline & & Spray angle & 21 & $\circ$ \\
\hline 04 & Biological Evaluation & Skin irritation test & \multicolumn{2}{|c|}{ No edema or redness of skin observed } \\
\hline
\end{tabular}

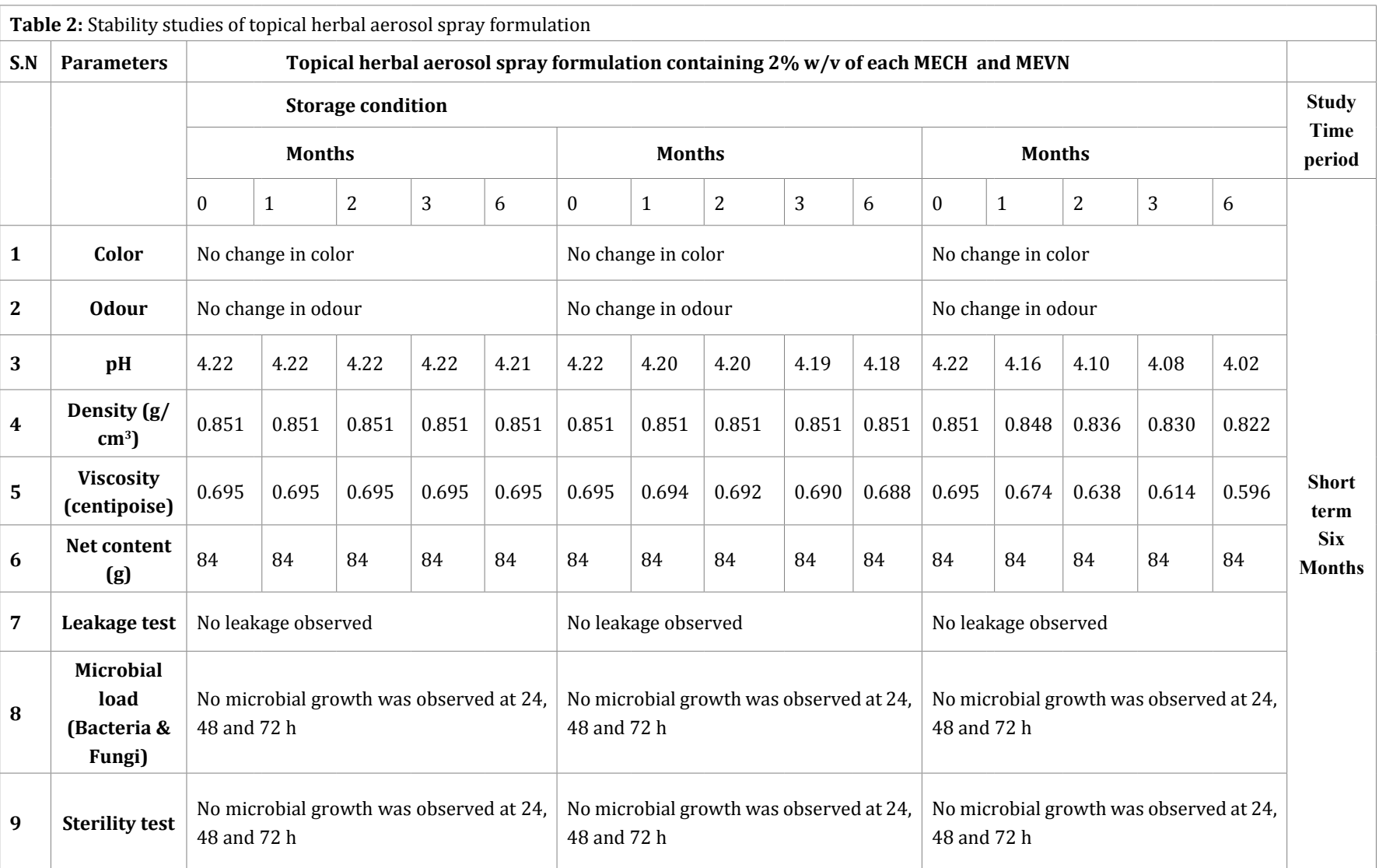




\section{Anti-Arthritic Activity}

\section{Body Weight}

The average gain/reduction in body weight was observed for all the groups after induction of arthritis in rats. Reduction in body weight was observed for arthritic control group whereas gain in body weight was observed in Diclofenac sodium treated (p.o.), MLECH and MLEVN treated groups, Diclofenac aerosol spray treated groups and topical herbal aerosol spray formulation treated groups, when compared with the normal group [Table 3]

\section{Paw Volume}

Alteration in rat paw volume was recorded on 25th, 29th, 35th and 42nd days in Table 4. The arthritic control groups showed signs of arthritis development as seen by increase in paw volume. Significant $(\mathrm{p}<0.01)$ reduction in rat paw volume was observed in Diclofenac sodium (p.o.), MLECH and MLEVN (p.o.), Diclofenac aerosol spray and herbal aerosol spray formulation treated groups, on 21st day after CFA induction [Table 4].

\begin{tabular}{|c|c|c|c|c|c|c|c|}
\hline Groups & $\begin{array}{c}\text { Initial body } \\
\text { weight (g) }\end{array}$ & $\begin{array}{c}\text { Body wt after } \\
22 \text { days of CFA } \\
\text { induction }\end{array}$ & $\begin{array}{c}\text { Body wt after } \\
\text { treatment } \\
25^{\text {th }} \text { day }\end{array}$ & $\begin{array}{c}\text { Body wt after } \\
\text { treatment } \\
29^{\text {th }} \text { day }\end{array}$ & $\begin{array}{c}\text { Body wt after } \\
\text { treatment } \\
35^{\text {th }} \text { day }\end{array}$ & $\begin{array}{c}\text { Body wt after } \\
\text { treatment } \\
42^{\text {nd }} \text { day }\end{array}$ & $\begin{array}{l}\text { Weight } \\
\text { gain (g) }\end{array}$ \\
\hline $\begin{array}{c}\text { Normal Control } \\
(1 \% \text { w/v SLS) }\end{array}$ & $146.26 \pm 1.47$ & $174.16 \pm 1.53$ & $171.50 \pm 0.84$ & $175.66 \pm$ c 0.88 & $182.00 \pm 1.03$ & $194.16 \pm 0.98$ & $\begin{array}{c}47.91 \pm \\
1.78\end{array}$ \\
\hline Arthritic control & $143.45 \pm 1.22$ & $158.30 \pm 1.25$ & $160.50 \pm 1.25$ & $161.66 \pm 1.08$ & $163.16 \pm 0.94$ & $164.33 \pm 1.17$ & $\begin{array}{c}20.88 \pm \\
2.15\end{array}$ \\
\hline $\begin{array}{c}\text { Diclofenac } \\
\text { sodium } 10 \mathrm{mg} / \\
\text { kg (p.o.) }\end{array}$ & $148.53 \pm 0.68$ & $160.66 \pm 0.84^{b}$ & $170.00 \pm 0.96^{\mathrm{b}}$ & $173.33 \pm 1.62^{b}$ & $180.5 \pm 0.56^{\mathrm{b}}$ & $186.33 \pm 0.61^{b}$ & $\begin{array}{c}38.30 \pm \\
1.08^{\mathrm{b}}\end{array}$ \\
\hline $\begin{array}{l}\text { MLECH } 400 \mathrm{mg} / \\
\mathrm{kg} \& \text { MLEVN } 400 \\
\mathrm{mg} / \mathrm{kg} \text { (p.o.) }\end{array}$ & $146.26 \pm 1.47$ & $155.00 \pm 1.06^{b}$ & $169.50 \pm 0.42^{b}$ & $172.83 \pm 0.30^{b}$ & $174.5 \pm 1.11^{\mathrm{b}}$ & $184.33 \pm 0.91^{b}$ & $\begin{array}{l}37.4 \pm \\
1.54^{\mathrm{b}}\end{array}$ \\
\hline $\begin{array}{c}\text { Diclofenac } \\
\text { topical aerosol } \\
\text { spray (8 mg/ } \\
\text { day) }\end{array}$ & $144.43 \pm 1.81$ & $154.50 \pm 1.28^{\mathrm{b}}$ & $174.33 \pm 1.66^{b}$ & $177.83 \pm 1.49^{b}$ & $\begin{array}{c}188.33 \pm \\
0.88^{\mathrm{b}}\end{array}$ & $193.50 \pm 0.61^{b}$ & $\begin{array}{l}51.6 \pm \\
1.14^{\mathrm{b}}\end{array}$ \\
\hline $\begin{array}{l}\text { Topical herbal } \\
\text { aerosol spray } \\
\text { formulation } \\
\text { (64 mg/day) }\end{array}$ & $143.16 \pm 1.01$ & $154.60 \pm 1.17^{b}$ & $171.00 \pm 0.73^{b}$ & $176.80 \pm 1.42^{b}$ & $\begin{array}{c}184.16 \pm \\
1.60^{\mathrm{b}}\end{array}$ & $187.00 \pm 1.13^{b}$ & $\begin{array}{c}42.56 \pm \\
2.01^{\mathrm{b}}\end{array}$ \\
\hline
\end{tabular}

Data provided as mean \pm SEM $(n=6)$; $\mathrm{b}<0.01$ treated groups vs. arthritic control

Table 4: Evaluation of anti-arthritic activity of MLECH, MLEVN and formulations in CFA induced arthritic rats

\begin{tabular}{|c|c|c|c|c|c|c|c|}
\hline \multirow{3}{*}{ Groups } & \multirow{3}{*}{ Dose } & \multicolumn{6}{|c|}{ Rat paw volume (mm) } \\
\hline & & \multicolumn{3}{|c|}{ Before treatment } & \multicolumn{3}{|c|}{ After treatment } \\
\hline & & Initial & After 21 days & $25^{\text {th }}$ day & $29^{\text {th }}$ day & $35^{\text {th }}$ day & $42^{\text {nd day }}$ \\
\hline Normal control & $1 \%$ w/v SLS (5 ml/kg) & $4.91 \pm 0.19$ & $5.26 \pm 0.23$ & $4.90 \pm 0.31$ & $5.25 \pm 0.17$ & $5.39 \pm 0.13$ & $5.52 \pm 0.16$ \\
\hline Arthritic control & $1 \%$ w/v SLS (5 ml/kg) & $4.98 \pm 0.30$ & $10.48 \pm 0.26$ & $10.75 \pm 0.10$ & $10.26 \pm 0.08$ & $10.41 \pm 0.07$ & $10.42 \pm 0.10$ \\
\hline Diclofenac sodium (p.o.) & $10 \mathrm{mg} / \mathrm{kg}$ & $5.33 \pm 0.16$ & $10.48 \pm 0.22$ & $9.36 \pm 0.07^{\mathrm{b}}$ & $8.93 \pm 0.09^{b}$ & $7.71 \pm 0.26$ & $6.91 \pm 0.32^{\mathrm{b}}$ \\
\hline MLECH + MLEVN & $400+400 \mathrm{mg} / \mathrm{kg}$ & $4.94 \pm 0.17$ & $10.62 \pm 0.33$ & $9.66 \pm 0.07^{b}$ & $8.04 \pm 0.21^{b}$ & $7.59 \pm 0.18^{b}$ & $7.30 \pm 0.16^{\mathrm{b}}$ \\
\hline $\begin{array}{l}\text { Diclofenac topical } \\
\text { aerosol spray }\end{array}$ & $8 \mathrm{mg} /$ day & $5.05 \pm 0.14$ & $10.41 \pm 0.21$ & $9.72 \pm 0.06^{\mathrm{b}}$ & $8.84 \pm 0.15^{b}$ & $7.71 \pm 0.15^{\mathrm{b}}$ & $7.55 \pm 0.17^{\mathrm{b}}$ \\
\hline $\begin{array}{l}\text { Topical herbal aerosol } \\
\text { spray formulation }\end{array}$ & $64 \mathrm{mg} /$ day & $4.87 \pm 0.18$ & $10.39 \pm 0.25$ & $9.98 \pm 0.15^{a}$ & $7.91 \pm 0.34^{\mathrm{b}}$ & $7.87 \pm 0.16^{\mathrm{b}}$ & $6.91 \pm 0.24^{\mathrm{b}}$ \\
\hline
\end{tabular}




\section{Arthritic Score}

The severity of arthritis was assessed by visual arthritic scoring systems described by Laird et al [31]. The arthritic test scores were assigned as shown in Table 5 revealed that the pain associated with CFA induced arthritis was significantly decreased in Diclofenac, MLECH \& MLEVN and topical herbal aerosol spray formulation treated groups. [Table 5]

\section{Hematological Parameters}

Rats treated with Diclofenac sodium (p.o.), MLECH and MLEVN, Diclofenac aerosol spray and herbal aerosol spray formulation elicited decrease in WBC count, ESR and increase in $\mathrm{Hb}$ count and RBC count, when compared with arthritic control groups [Table 6].

\section{Biochemical Parameters}

Significant $(\mathrm{p}<0.01)$ decrease in urea and uric acid concentration was observed in Diclofenac sodium treated (p.o.), MLECH and MLEVN treated groups, Diclofenac aerosol spray treated groups and herbal aerosol spray formulation treated groups, when compared with the arthritic control group [Table $6]$.

\section{Serum Biomarkers}

\section{In Vitro Determination of CRP}

The serum of the tested animal groups before treatment showed agglutination and no agglutination was observed for the serum of groups treated with Diclofenac sodium (p.o.), MLECH and MLEVN (p.o.), Diclofenac topical aerosol spray and topical herbal aerosol spray formulation.

\section{In Vitro Determination of Rheumatic Factor}

$\mathrm{RF}$ is the most useful prognostic marker for the diagnosis of rheumatoid arthritis, agglutination is used because greater sensitivity and simplicity. No agglutination was observed in the serum of the tested animal groups the treatment of Diclofenac sodium (p.o.), MLECH and MLEVN (p.o.), Diclofenac topical aerosol spray and topical herbal aerosol spray formulation.

\section{In Vitro Determination of TNF-A}

TNF $\alpha$ level in arthritic induced rats was found to be 44.90 $\mathrm{pg} / \mathrm{ml}$. The TNF $\alpha$ level of Diclofenac treated (p.o.), MLECH and MLEVN treated, Diclofenac topical aerosol spray treated and topical herbal aerosol spray formulation treated groups found to be less than the arthritic induced rats. Thus this study revealed that Diclofenac treated (p.o), MLECH and MLEVN treated, Diclofenac topical spray treated and topical herbal aerosol spray formulation treated groups reduced the levels of TNF $\alpha$ [Table 7].

\begin{tabular}{|c|c|c|c|c|}
\hline \multirow{2}{*}{ Groups } & \multicolumn{2}{|c|}{ Pain test } & \multirow{2}{*}{ Mobility score } & \multirow{2}{*}{ Stance score } \\
\hline & Extension & Flexion & & \\
\hline Arthritic control & $9.66 \pm 0.21$ & $8.50 \pm 0.34$ & $1.16 \pm 0.16$ & $1.33 \pm 0.21$ \\
\hline Diclofenac $10 \mathrm{mg} / \mathrm{kg}$ p.o. & $4.33 \pm 0.21^{\mathrm{b}}$ & $3.83 \pm 0.16^{\mathrm{b}}$ & $2.83 \pm 0.16^{\mathrm{b}}$ & $3.16 \pm 0.16^{\mathrm{b}}$ \\
\hline MLECH 400 + MLEVN 400 p.o. & $6.16 \pm 0.16^{\mathrm{b}}$ & $4.16 \pm 0.16^{b}$ & $2.16 \pm 0.16^{\mathrm{a}}$ & $2.50 \pm 0.22^{\mathrm{b}}$ \\
\hline $\begin{array}{l}\text { Diclofenac topical aerosol spray (8 } \\
\mathrm{mg} / \text { day) }\end{array}$ & $4.50 \pm 0.22^{\mathrm{b}}$ & $3.50 \pm 0.34^{\mathrm{b}}$ & $3.00 \pm 0.36^{\mathrm{b}}$ & $2.83 \pm 0.16^{\mathrm{b}}$ \\
\hline $\begin{array}{l}\text { Topical herbal aerosol spray } \\
\text { formulation } \quad(64 \mathrm{mg} / \text { day })\end{array}$ & $5.50 \pm 0.22^{\mathrm{b}}$ & $4.33 \pm 0.21^{b}$ & $2.33 \pm 0.33^{\mathrm{a}}$ & $2.10 \pm 0.16^{\mathrm{b}}$ \\
\hline
\end{tabular}

\begin{tabular}{|c|c|c|c|c|c|c|c|c|c|c|c|c|}
\hline \multirow[b]{2}{*}{ Groups } & \multicolumn{2}{|c|}{$\mathrm{Hb}(\mathrm{mg} \%)$} & \multicolumn{2}{|c|}{$\mathrm{WBC}\left(\mathrm{x}^{\left.10^{3} / \mathrm{mm}^{3}\right)}\right.$} & \multicolumn{2}{|c|}{$\operatorname{RBC}\left(x 10^{6} / \mathrm{mm}^{3}\right)$} & \multicolumn{2}{|c|}{$\operatorname{ESR}(\mathbf{m m} / \mathbf{h})$} & \multicolumn{2}{|c|}{ Before treatment } & \multicolumn{2}{|c|}{ After treatment } \\
\hline & $\begin{array}{c}\text { Before } \\
\text { treatment }\end{array}$ & $\begin{array}{c}\text { After } \\
\text { treatment }\end{array}$ & $\begin{array}{c}\text { Before } \\
\text { treatment }\end{array}$ & $\begin{array}{c}\text { After } \\
\text { treatment }\end{array}$ & $\begin{array}{c}\text { Before } \\
\text { treatment }\end{array}$ & $\begin{array}{c}\text { After } \\
\text { treatment }\end{array}$ & $\begin{array}{c}\text { Before } \\
\text { treatment }\end{array}$ & $\begin{array}{c}\text { After } \\
\text { treatment }\end{array}$ & $\begin{array}{c}\text { Urea (mg/ } \\
\text { dl) }\end{array}$ & $\begin{array}{l}\text { Uric acid } \\
(\mathrm{mg} / \mathrm{dl})\end{array}$ & $\begin{array}{c}\text { Urea (mg/ } \\
\text { dl) }\end{array}$ & $\begin{array}{l}\text { Uric acid } \\
(\mathrm{mg} / \mathrm{dl})\end{array}$ \\
\hline $\begin{array}{l}\text { Normal } \\
\text { control }\end{array}$ & $\begin{array}{c}15.56 \pm \\
0.27\end{array}$ & $\begin{array}{c}16.13 \pm \\
0.15\end{array}$ & $\begin{array}{c}6.61 \pm \\
0.27\end{array}$ & $\begin{array}{c}6.78 \pm \\
0.06\end{array}$ & $\begin{array}{c}9.71 \pm \\
0.22\end{array}$ & $\begin{array}{c}10.26 \pm \\
0.15\end{array}$ & $\begin{array}{c}4.83 \pm \\
1.47\end{array}$ & $5.00 \pm 0.63$ & $16.16 \pm 0.91$ & $\begin{array}{c}2.66 \pm \\
0.09\end{array}$ & $\begin{array}{c}16.60 \pm \\
0.91\end{array}$ & $2.86 \pm 0.07$ \\
\hline $\begin{array}{c}\text { Arthritic } \\
\text { control }\end{array}$ & $10.9 \pm 0.17^{\mathrm{a}}$ & $\begin{array}{c}10.26 \pm \\
0.10\end{array}$ & $\begin{array}{c}11.78 \pm \\
0.32^{\mathrm{a}}\end{array}$ & $\begin{array}{c}10.2 \pm \\
0.2\end{array}$ & $7.66 \pm 0.30^{\mathrm{a}}$ & $\begin{array}{c}5.67 \pm \\
0.20\end{array}$ & $\begin{array}{c}36.16 \pm \\
1.19^{\mathrm{a}}\end{array}$ & $\begin{array}{c}40.33 \pm \\
1.43\end{array}$ & $44.35 \pm 1.50^{\mathrm{a}}$ & $\begin{array}{l}6.96 \pm \\
0.16^{\mathrm{a}}\end{array}$ & $\begin{array}{c}44.8 \pm \\
3.62\end{array}$ & $7.15 \pm 0.13$ \\
\hline $\begin{array}{c}\text { Diclofenac } \\
\text { sodium (10 } \\
\text { mg/kg) }\end{array}$ & $\begin{array}{c}11.01 \pm \\
0.03^{\mathrm{a}}\end{array}$ & $\begin{array}{c}15.91^{ \pm} \\
0.21^{\mathrm{b}}\end{array}$ & $\begin{array}{c}11.95^{ \pm} \\
0.35^{\mathrm{a}}\end{array}$ & $\begin{array}{l}7.53 \pm \\
0.17^{\mathrm{b}}\end{array}$ & $\begin{array}{l}7.23 \pm \\
0.19^{\mathrm{a}}\end{array}$ & $\begin{array}{l}9.01 \pm \\
0.23^{\mathrm{b}}\end{array}$ & $\begin{array}{c}33.5 \pm \\
1.17^{\mathrm{a}}\end{array}$ & $\begin{array}{c}13.66 \pm \\
1.11^{\mathrm{b}}\end{array}$ & $43.75 \pm 1.49^{\mathrm{a}}$ & $\begin{array}{l}7.20 \pm \\
0.11^{\mathrm{a}}\end{array}$ & $25.93 \pm 1.25^{\mathrm{b}}$ & $3.43 \pm 0.16^{\mathrm{b}}$ \\
\hline
\end{tabular}




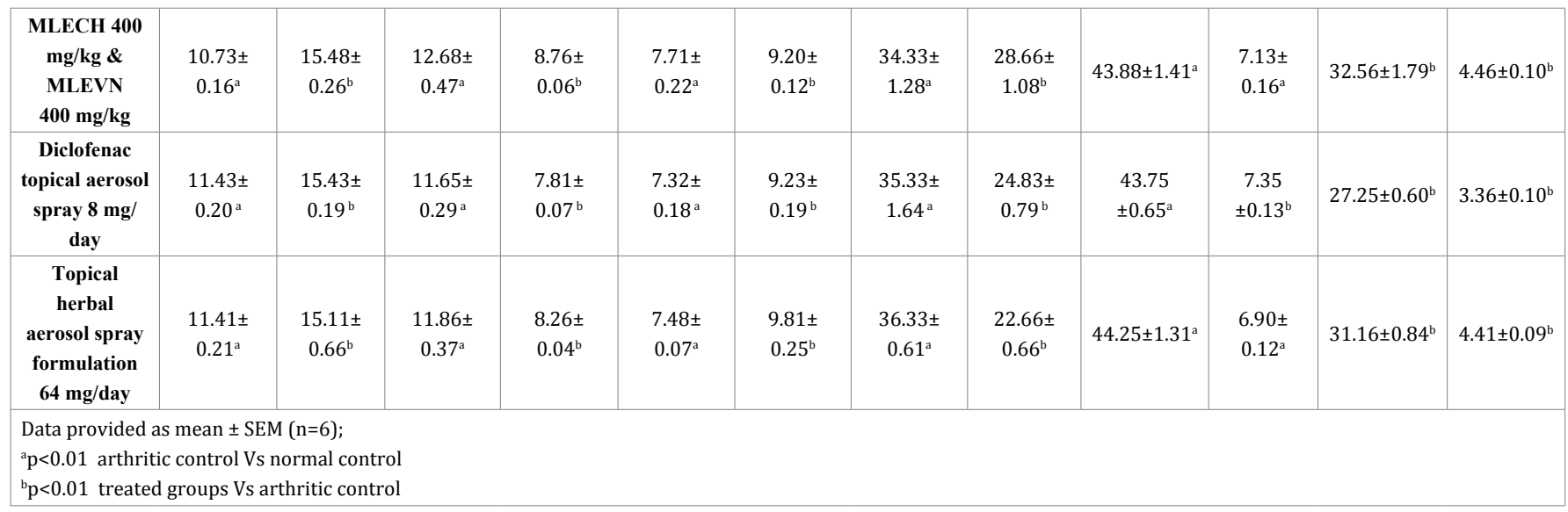

Table 7: In vitro determination of serum TNF $\alpha$ and interleukin levels in CFA induced arthritic rats

\begin{tabular}{|c|c|c|c|}
\hline \multirow{2}{*}{ Groups } & \multirow{2}{*}{$\mathrm{TNF} \alpha(\mathrm{pg} / \mathrm{ml})$} & \multicolumn{2}{|c|}{ Interleukin levels (pico $\mathrm{g} / \mathrm{ml}$ of serum) } \\
\hline & & IL-1ß & IL-6 \\
\hline Normal control & $1.36 \pm 0.23$ & $1.38 \pm 0.24$ & $19.58 \pm 1.14$ \\
\hline Arthritic control & $44.90 \pm 2.36^{\mathrm{a}}$ & $67.70 \pm 1.09^{\mathrm{a}}$ & $728.61 \pm 31.99^{a}$ \\
\hline Diclofenac sodium $10 \mathrm{mg} / \mathrm{kg}$ (p.o.) & $2.36 \pm 0.45^{b}$ & $23.34 \pm 1.34^{\mathrm{b}}$ & $323.33 \pm 16.55^{\mathrm{b}}$ \\
\hline $\begin{array}{c}\text { MLECH } 400 \mathrm{mg} / \mathrm{kg}+\text { MLEVN } 400 \mathrm{mg} / \\
\operatorname{kg} \text { (p.o.) }\end{array}$ & $29.04 \pm 0.63^{b}$ & $45.11 \pm 1.08^{\mathrm{b}}$ & $512.72 \pm 24.87^{b}$ \\
\hline $\begin{array}{c}\text { Diclofenac topical aerosol spray (8 } \\
\mathrm{mg} / \text { day) }\end{array}$ & $6.28 \pm 0.71^{\mathrm{b}}$ & $20.99 \pm 0.60^{\mathrm{b}}$ & $313.95 \pm 14.66^{b}$ \\
\hline $\begin{array}{l}\text { Topical herbal aerosol spray } \\
\text { formulation ( } 64 \mathrm{mg} / \text { day) }\end{array}$ & $19.79 \pm 0.81^{\mathrm{b}}$ & $36.55 \pm 1.25^{\mathrm{b}}$ & $428.12 \pm 10.17^{\mathrm{b}}$ \\
\hline \multicolumn{4}{|c|}{$\begin{array}{l}\text { All the values are mean } \pm \text { SEM }(n=6) \text {. } \\
{ }^{a} p<0.01 \text { arthritic control Vs normal control } \\
{ }^{b} p<0.01 \text {, treatment groups Vs arthritic control }\end{array}$} \\
\hline
\end{tabular}

\section{In Vitro Determination Of IL 1ß And IL 6}

Cytokines, IL $1 ß$ and IL 6 play a key role in mediating both acute and chronic inflammation and hence In vitro determination of IL- $1 ß$ and IL- 6 was determined for the serum of all the groups used in the arthritic experiment (Table 7). The cytokine levels was found to be increased in arthritic control, which was significantly $(\mathrm{p}<0.01)$ reduced in Diclofenac sodium treated (p.o.), MLECH and MLEVN treated groups, Diclofenac aerosol spray treated groups and herbal aerosol spray formulation treated groups.

\section{Photographic and Radiographic Analysis}

Representative photographs and radiographs (Figures1A and 1B) of trasotibial joint swelling of the left hind paws of rats from different groups were taken on 42 nd day. The soft tissue swelling around the joints observed in the arthritic control rats was significantly reduced in the Diclofenac sodium treated (p.o.), MLECH and MLEVN treated, Diclofenac topical aerosol spray treated and topical herbal aerosol spray formulation treated groups. [Figures 2A and 2B]

\section{Spleen and Thymus Weight}

The weights of spleen and thymus recorded after sacrificing the rats on 42nd day. Significant $(\mathrm{p}<0.01)$ reduction of spleen and thymus weight was observed for the Diclofenac sodium treated (p.o.), MLECH and MLEVN treated groups, Diclofenac aerosol spray treated groups and herbal aerosol spray formulation treated groups when compared with the arthritic control group.

\section{Histopathological Studies}

RHistological examination of normal specimen of joint showed normal joint space, normal adjacent soft tissue and synovium and cartilage. CFA induced specimen of joint, showed adjacent soft tissue and synovial hyperplasia with dense inflammation in the soft tissue around the joint. Diclofenac (p.o.) treated specimen joint exhibited occasional inflammatory cells \& hyperplasia of the synovium when compared with CFA treated groups. Specimen of joint, orally treated with MLECH, MLEVN showed mild inflammation \& hyperplasia of the synovium when compared with CFA treated groups and Diclofenac (p.o.) treated groups. Specimen of joint of Diclofenac topical aerosol spray treated groups showed moderate inflammation \& hyperplasia of the synovium. Specimen of joint of topical herbal aerosol spray formulation treated groups showed occasional inflammatory cells and hyperplasia of the synovium when compared with CFA and Diclofenac aerosol spray treated groups. 


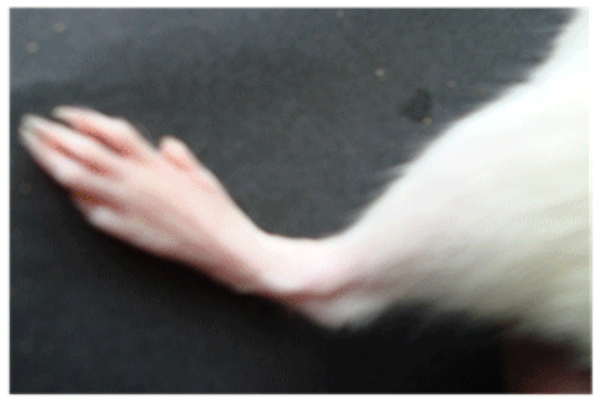

Normal control(a)

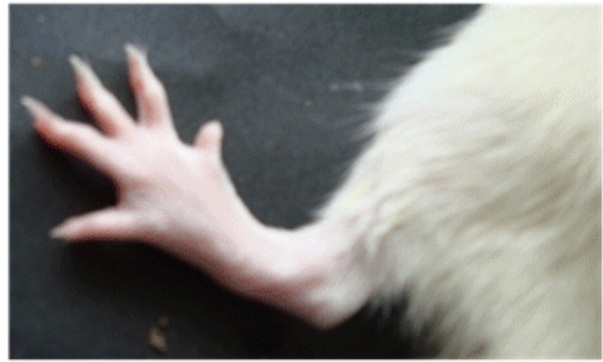

CFA Control(b)

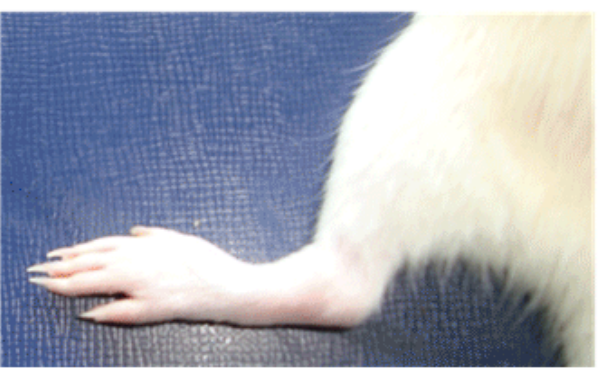

CFA and diclofenac

Treated (p.o) (c)

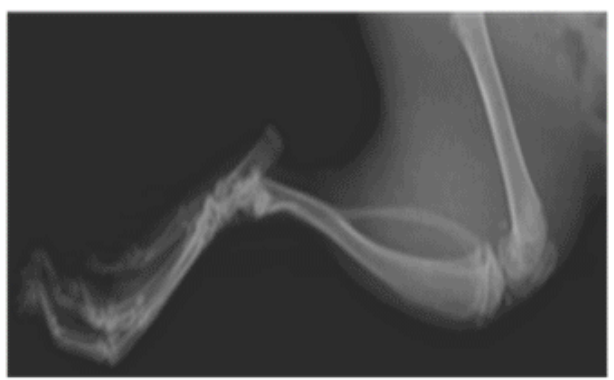

Normal control showing no soft Tissue swelling around the joints (a)

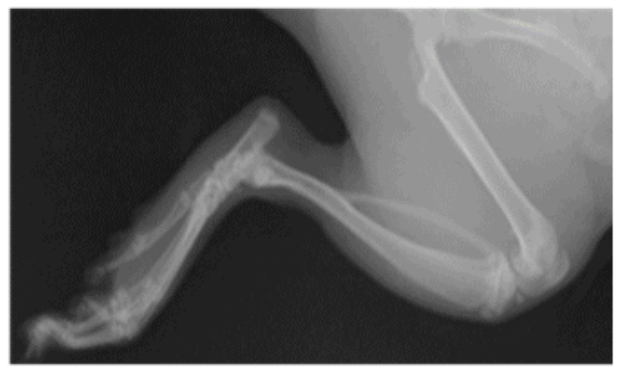

CFA Control showing soft tissue swelling around the joints (b)

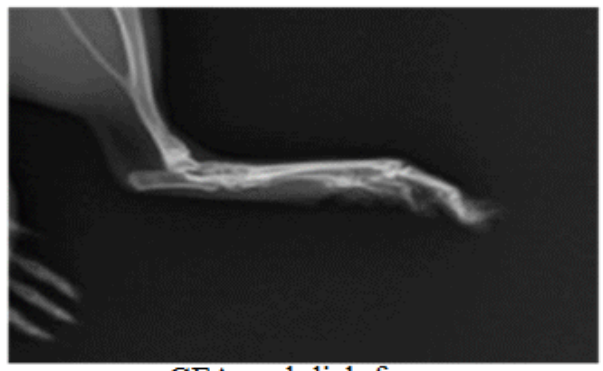

CFA and diclofenac

Treated (p.o) (c)

Figure 2A: (a) Photographic and radiographic analysis of normal rats. (b) Photographic and radiographic analysis of the same left hind paw taken 21 days after CFA injection (c) Photographic and radiographic analysis of the same left paw of diclofenac treated (p.o.) taken 21 days after CFA injection

\section{Discussion}

Cardiospermum halicacabum and Vitexnegundo individually reported to produce beneficial effects on RA [34-35].The phytoconstituents, luteolin and apigenin were reported to be present in leaf extract of $\mathrm{CH}$ and $\mathrm{VN}$ and they were isolated by Srinivas et al [5] and Achari et al [20]. Further these flavonoid aglycones reported to be present in these plants can penetrate the human skin [36] and exhibited in vivo anti-inflammatory activity via topical and oral routes of administration [37].The above findings, prompted us to formulate the topical aerosol spray using MLECH and MLEVN for the treatment of arthritis. Aluminium containers were used for packing the aerosol formulation as they withstand pressure up to $180 \mathrm{psig}$, temperature up to $130^{\circ} \mathrm{F}$, less cost, light weight, compatible with the active ingredients of the formulation, greater resistance to corrosion and it is good for light sensitive drugs, Mixture of hydrocarbons viz. propane and butane was used as propellant as they are less toxic, less reactive, less susceptible to hydrolysis and their environmental acceptance. After filling the product concentrate the containers were heated to $130^{\circ} \mathrm{F}$ and tested for leakage and labeled.

Quality control test performed for topical herbal aerosol spray formulation complied with USP standards and the stability study performed for 6 months for the formulated topical herbal aerosol spray was found to be stable and complied with ICH guidelines.

In our earlier acute oral toxicity study, carried out separately for MLECH and MLEVN was found to be safe upto a dose of 2000 $\mathrm{mg} / \mathrm{kg}$ and hence $1 / 20^{\text {th }}, 1 / 10^{\text {th }}$ and $1 / 5^{\text {th }}$ dose of $2000 \mathrm{mg} / \mathrm{kg}$ viz.100, 200, $400 \mathrm{mg} / \mathrm{kg}$ were used for anti-arthritic activity study. It was also observed from our earlier study, that the combination $(100+100,200+200,400+400 \mathrm{mg} / \mathrm{kg})$ of MLECH and MLEVN showed significant inhibition of inflammation in a dose dependent manner than the individually administered 


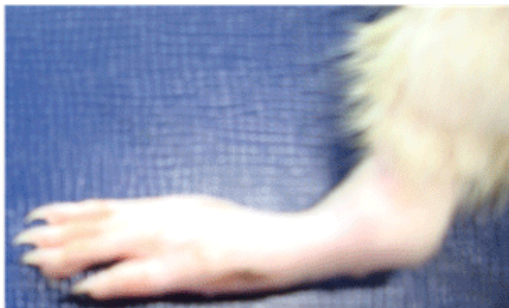

CFA and MLECH + MLEVN treated (p.o) (d)

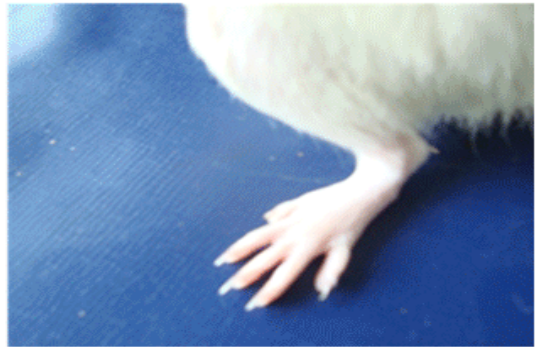

CFA and diclofenac aerosol treated (e)

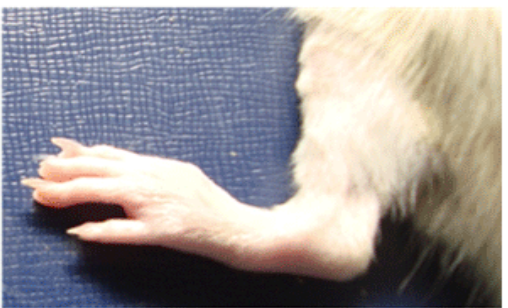

CFA and herbal aerosol formulation treated (f)

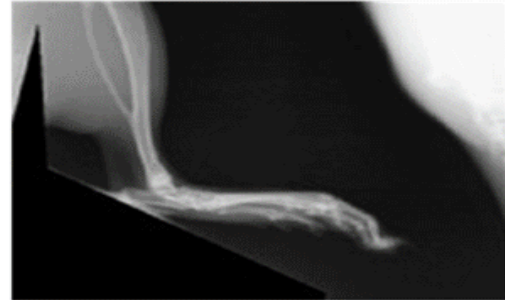

CFA and MLECH + MLEVN treated (p.o) showing no soft tissue swelling around the joints (d)

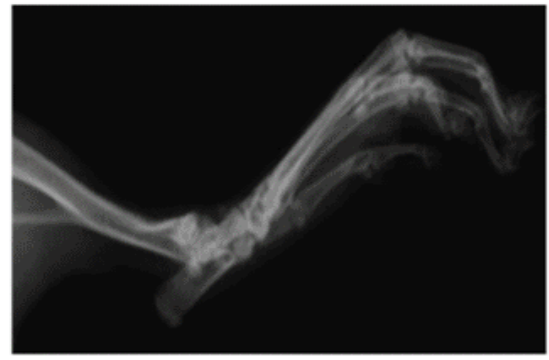

CFA and diclofenac aerosol treated showing no soft tissue swelling around the joints (d (e)

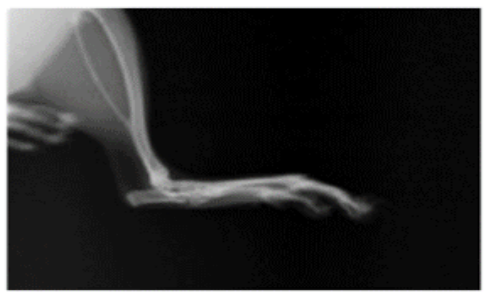

CFA and herbal aerosol formulation treated showing no tissue swelling around the joints (d (f)

Figure 2B: (d) Photographic and radiographic analysis of the same left hind paw of MLECH and MLEVN treated (p.o.) taken 21 days after CFA injection (e) Photographic and radiographic analysis of the same left hind paw of diclofenac topical aerosol spray treated taken21 days after CFA injection (f) Photograph and radiographic analysis of the same left hind paw of Topical herbal aerosol spray formulation taken 21 days after CFA injection.

doses of MLECH $(100,200,400 \mathrm{mg} / \mathrm{kg})$ and MLEVN $(100,200$ $400 \mathrm{mg} / \mathrm{kg}$ ). Based on these observations, each $400 \mathrm{mg} / \mathrm{kg}$ dose of MLECH and MLEVN was used for anti-arthritic study.

CFA-induced arthritis is the most widely used chronic test model which is associated with an immune-mediated inflammatory reaction and rat is unique in developing polyarthritis after CFA treatment and hence they were used for the study [38]. In first phase, irritant nature of the adjuvant induces inflammation and hence measurement of 3 to 5 day effect of treatment represents the efficacy on inflammation of primary lesions such as edema and soft-tissue thickening. The second phase, reduction in body weight due to immunogenic responses [39].

In the present study, administration of Diclofenac sodium, MLECH and MLEVN $(400+400 \mathrm{mg} / \mathrm{kg}$, p.o.), topical Diclofenac topical aerosol spray and topical herbal aerosol spray formulation in rats significantly reduced the progression of RA when compared with arthritic rats. Significant alterations in flexion pain test score, mobility score and stance score was observed for all the treated group of rats when compared with arthritic control rats. This alteration of arthritic test scores support the anti-arthritic activity of the MLECH \& MLEVN and topical herbal aerosol spray formulation.

Information related to the pathology of arthritis was obtained during the study from body weight changes and hematological parameters. Restoration of body weight of rats was observed after treatment of MLECH and MLEVN and topical herbal aerosol spray formulation. Decrease in body weight during inflammation in arthritic control group was reported [40] due to deficient absorption of nutrients through intestine. It was reported that 
treatment of anti-inflammatory drugs normalizes the process of absorption and hence the restoration of body weight in MLECH, MLEVN and Diclofenac sodium treated groups may involve improvement of intestinal absorption of nutrients and reduction in the distress caused by the severity of arthritis. It has been reported that a moderate rise in the WBC count occurs in arthritic conditions due to an IL-1B mediated rise in the respective colony stimulating factors. The present study reveals that MLECH, MLEVN and Diclofenac sodium treatments tend to normalize the WBC count. Characteristic hematological alterations such as increased $\mathrm{Hb}$ count and decreased ESR by MLECH and MLEVN support the anti-arthritic effect. It is proposed that the reduction in the $\mathrm{Hb}$ count during arthritis results from reduced erythropoietin levels, a decreased response of the bone marrow erythropoietin and premature destruction of red blood cells and increase in ESR is due to formation of endogenous protein, globulin and fibrinogen [41]. Increase in serum levels of CRP, RF37 TNF- $\alpha$, IL1 $\beta$ and IL6 are characteristic feature of RA [42] and hence, in vitro determination of serum biomarkers such as CRP, RF, TNF- $\alpha$, IL1 $\beta$ and IL6 were performed for the arthritic control and all the treated groups. Significant reduction of serum biomarker levels in treated rats when compared with arthritic control rats support the anti-arthritic activity.

Radiological observations of the rats provided the evidence for the inhibition of arthritis after treatment of Diclofenac sodium p.o, MLECH and MLEVN (400+400 mg/kg, p.o.), topical Diclofenac topical aerosol spray and topical herbal aerosol spray formulation.

Synovial Inflammation and hyperplasia is considered to be the important pathological condition of rheumatoid arthritis [43]. Histological examination of joint showed significant reduction of inflammation in treated group of rats when compared with arthritic control rats.

Significant reduction in thymus and spleen weight of rats after treatment with MLECH \& MLEVN and topical herbal aerosol spray formulation support the anti-arthritic activity.

\section{Conclusion}

Thus from our study results, we conclude that the developed topical herbal aerosol spray formulation elicited anti-arthritic activity which may be due to the presence of luteolin and apigenin in MLECH and MLEVN respectively.

\section{Acknowledgements}

The authors thank Department of Science and Technology, Govt of India, New Delhi whole heartedly for providing funds to carry out this research. The authors are also thankful to Kovai Medical Center Research and Educational Trust, Kovai Estate Kalapatti Road, Coimbatore, Tamilnadu for providing facilities necessary for carrying out the work.

\section{Declaration of interest}

The authors report no declaration of interest.

\section{References}

1. Bernatsky S, ErhmannFeldmann D. Discontinuation of methotrexate therapy in older patients with newly diagnosed rheumatoid arthritis. Analysis of administrative health data bases in Quebec, Canada. Drugs ageing. 2008;25:879-884.

2. Vonkeman HE, Van De Laar MA. Nonsteroidal anti-inflammatory drugs: adverse effects and their prevention.Semin Arthritis Rheum. 2010;39[4]:294-312.

3. Gambaro G, Perazella MA. Adverse renal effects of anti-inflammatory agents. Evaluation of selective and nonselective cyclooxygenase inhibitors. J Intern Med. 2003;253:643-652.

4. Weinblatt ME, Dixon IA, Falchuk KR. Serious liver disease in a patient receiving methotrexate and leflunomide. Arthritis Rheum.2000;43(11):2609-2611.

5. Srinivas K, Choudary KA, Rao SS, SatyanarayanaT, Krishna Rao RV. Phytochemical examination of Cardiospermumhalicacabum Linn. Indian J Nat Prod.1998; 14:24-27.

6. Newmaster SG, Ragupathy S, Ivanhoff RF, Nirmala CB. Mechanisms of ethnobiological classifications. Ethnobotany.2006b;18:4-26.

7. Eswar Kumar K, Mastan SK, Amrander Reddy G, Ragunandan N, Sreekanth N, Chaitanya G. Anti-arthritic property of the ethanolic leaf extracts of Cardiospermum halicacabumLinn. Biomed Pharmacol J. 2008;1:2-4.

8. Jeyadevi R, Sivasudha T, Rameshkumar A, Dinesh Kumar L. Antiarthritic activity of Indian leafy vegetable Cardiospermum halicacabum in Wistar rats and UPLC- QTOF-MS/MS identification of the putative active phenolic compounds. Inflamm Res.2011;62(1):115-126.

9. Muthu C, Ayyanar M, Raja N, Ignacimuthu S. Medicinal plants used by traditional healers in Kanchipuram district of Tamilnadu. India. J. Ethnobiol. Ethnomed. 2006;2:43-46.

10. Sheeba MS, Asha VV. Cardiospermum halicacabum ethanol extract inhibits LPS induced COX-2, TNF-alpha and iNOS expression, which is mediated by NF-kappa B regulation, in RAW264.7 cells. J Ethnopharmacol. 2009;124:39-44.

11.Wang J, Wang N, Yao X, Kitanakas RIS. Inhibitory activity of Chinese herbal medicines towards histamine release from mast cells and nitric oxide production by macrophage like cell line. J Nat Med. 2006;60:7377.

12. Kumaran A, Karunakaran RJ. Antioxidant activities of the methanol extract of Cardiospermum halicacabum. Pharm Biol.2006;44:146151.

13.Gopalakrishnan C, Dhananjayan R, Kameswaran L. Studies on the pharmacological actions of Cardiospermum halicacabum. Indian J Physiol and Pharmacol.1976;20: 203-206.

14.Asha VV, Pushpangadan P. Antipyretic activity of Cardiospermum halicacabum. Indian J. Exp Biol. 1999;37:411-414.

15. Waako PJ, Gumede B, Smith P, Folb PI. The in vitro and in vivo antimalarial activity of Cardiospermum halicacabumL. and Momordica foetida Schumch. Et Thonn.J Ethnopharmacol. 2005;99:137-143.

16. Santakumari G, Pillai NR, Nair RB. Diuretic activity of Cardiospermum halicacabumLinn in rats. J Sci. Res. Plant Med.1981; 2:32-35.

17.VenkatRao N, Chandraprakash K, Shanta Kumar SM. Pharmacological investigation of Cardiospermumhalicacabum (Linn) in different animal models of diarrhoea. Indian J Pharmacol.2006; 38(5):346-49; DOI: 10.4103/0253-7613.27703.

18. Veeramani C, Pushpavalli G, Pugalendi KV. Antihyperglycaemic effect of Cardiospermumhalicacabum Linn. Leaf extract on STZ-induced diabetic rats. J Appl. Biomed.2008; 6:19-26. 
19. Sheeba MS, Asha VV. Effect of Cardiospermumhalicacabumon ethanol induced gastric ulcers in rats.J Ethnopharmacol.2006; 106(1):105110. DOI:10.1016/j.jep.2005.12.009.

20. Achari B, Chowdhury US, Dutta PK, Pakrashi SC. Two isomeric flavonones from Vitexnegundo. Phytochemistry. 1984; 23(3): 703-704. doi.org/10.1016/S0031-9422(00)80420-3

21. Dharmasiri MG, Jayakody JARC, Galhena G, Liyanage SSP, Ratnasooryia WD: Anti-inflammatory and analgesic activities of mature fresh leaves of Vitexnegundo. J Ethnopharmacol. 2003;87(2-3):199-206.

22. Tamhankar CP, Saraf MN. Anti-arthritic activity of V. negundo L. Ind J Pharm Sci. 1994; 56:154-160.

23. Gupta M, Mazumder UK, Bhawal S. CNS activity of Vitexnegundo Linn in mice. Indian J Exp Biol. 1999;37(2):143-146.

24. Avadhoot V, Rana AC. Hepatoprotective effect of Vitexnegundoagainst carbon tetrachloride-induced liver damage. Arch Pharm Res. 1991;14(1): 96-98.

25. Adnaik RS, Pai PT, Mulem SN, Naikwade NS, Magdum CS. Laxative Activity of VitexnegundoLinn Leaves Asian J. Exp Sci. 2008;22(1):159160.

26. Nair AM, Saraf MN. Inhibition of antigen and compound 48/80 induced contraction of guinea pig trachea by ethanolic extract of the leaves of Vitexnegundo Linn. Indian J Pharmacol.1995; 27(4):230- 233.

27. Guha G, Rajkumar V, Ashok KumarR. Polyphenolic constituents of methanolic and aqueous extracts of Vitexnegundo render protection to Hep3B cells against oxidative cytotoxicity. Food ChemToxicol. 2010;48(8-9):2133-138. doi:10.1016/j.fct.2010.05.017.

28. Lachman L, Liberman HA, Kanig JL. The Theory \& Practice of Industrial Pharmacy, third ed. Varghese Publishers.

29. USP. Physical test and determination United States Pharmacopoeia. United States Pharmacopoeial convention INC, Asian ed. Rockville. 1990;1556-57.

30. Mizushima Y, Tsukada W, Akimoto T. A Modification of rat adjuvant arthritis for testing anti-rheumatic drugs. J Pharm Pharmacol. 1972; 24(10):781-785. doi:10.1111/j.2042-7158.1972.tb08882.x

31. Laird JMA, Carter AJ, Grauert M, Cervero F. Analgesic activity of a novel use-dependent sodium channel blocker, crobenetine, inmonoarthritic rats. Br J Pharmacol. 2001;134(8):1742-1748. doi:10.1038/ sj.bjp.0704428
32. Mangesh B, Virendra GK, Rohini RP, Vivek BP, Hardik H. Therapeutic effect of polyherbal preparation on adjuvant induced arthritis in Wistar rats. Int J Pharm Sci. 2011; 3(2):186-192.

33. Murphy CT, McCarroll SA, Bargmann CI, Fraser A, Kamath RS, Ahringer $\mathrm{J}$, et al. Genes that act downstream of DAF-16 to influence the lifespan of Caenorhabditiselegans. Nature. 2003; 424(6946):277-283; Doi:10.1038/nature01789

34. Ragupathy S, Newmaster SG, GopinadhanP, Candice BN. Exploring Ethnobiological Classifications for Novel Alternative Medicine: A case study of Cardiospermumhalicacabum L. (Modakathon, Balloon Vine) as a traditional herb for treating rheumatoid arthritis. Ethnobotany.2007;19(1-2):1-18.

35. Renukadevi P, Krishna Kumari S, Kokilavani C. Effect of Vitexnegundo leaf extract on the free radicals scavengers in complete Freund's adjuvant induced arthritic rats. Indian J ClinBiochem.2007;22:143-147.

36. Giinter S, Irmargd M, Ute W, Chistoph MS. Anti carcinogenic effects of the flavonoid luteolin. Molecules.2008; 13(10): 2628-2651.

37.Song JL, Kun HS, Hyeun WC, Jae CD. Keun YJ, Sam SK, et al. Antiinflammatory activity of naturally occurring flavone and flavonol glycosides. Arcd Pharm Res.1993; 16:25-28.

38. Nielen MM, Schaardenburg DV, Reesink HW, Twisk JWR, Van De Stadt RJ, VanderHorst BIE, et al. Simultaneous development of acute phase response and auto antibodies in preclinical Rheumatoid arthritis. Ann Rheum Dis.2006; 65(4): 535-537; Doi: 10.1136/ard.2005.040659

39. Singh B, Bani S, Gupta DK, Chandan BK, Kaul A. Anti-inflammatory activity of 'TAF': an active fraction from the plant Barleriaprionitis Linn. J Ethnopharmacol.2003; 85(2-3):187-193.

40.Patil KS, Suryavanshi J. Effect of Celastrucpaniculatus Wild seed on adjuvant induced arthritis in rats.Phcog Mag.2007; 3(11):177-181.

41. Hu F, Hepburn HR, Li Y, Chen M, Randloff SE, Patil MJ. Effect of ethanol and water extracts of propolis (bee glue) on acute inflammatory animal models. J Ethnopharmacol.2005; 100(3):276-83; DOI:10.1016/j. jep.2005.02.044

42. Feldmann M, Maini SR. Role of cytokines in rheumatoid arthritis an education in pathophysiology and therapeutics. Immunol Rev.2008; 223:7-19; Doi: 10.1111/j.1600-065X.2008.00626.x

43. Firestein GS: Invasive fibroblast-like synoviocytes in rheumatoid arthritis. Passive respondent or transformed aggressors? Arthritis Rheum.1996; 39(11):1781-1790. 